\title{
Stabilization of time delayed nonnegative polynomial systems through kinetic realization*
}

\author{
György Lipták $^{1}$ and Katalin M. Hangos ${ }^{1,2}$ and Gábor Szederkényi ${ }^{1,3}$
}

\begin{abstract}
A new stabilizing feedback design method is proposed in this paper for time delayed nonnegative polynomial systems with a linear input structure. Using a polynomial state feedback, the open loop system is transformed into a complex balanced kinetic system that is known to be stable with a known Lyapunov-Krasovskii functional. The computation problem is solved through semidefinite programming exploiting the fact that the reaction graph structure and weighting of a kinetic polynomial system are non-unique.
\end{abstract}

\section{INTRODUCTION}

It is known that important dynamical phenomena occurring in real world can often be explained in a satisfactory way by using time delays in the equations [10], [12], [11]. However, differential equations with time delay can produce much more complicated dynamics than ordinary differential equations in the general case. For example, a time delay may cause a stable equilibrium to become unstable, even if the dynamics is linear. Therefore, sophisticated methods are needed for dynamic analysis and control design for time delay systems. For example, it is reported that stabilizing of polynomial time delay systems can be solved by using Lyapunov-Krasovskii functional and sum of squares decomposition [22], [23].

On the other hand, several kinds of dynamical phenomena in nature or technology can be modelled by the class of nonnnegative systems, that are dynamical systems with nonnegative state variables. These include e.g. biochemical reaction networks, population dynamics, a wide range of process systems, and certain economical or transportation processes [26], [28], [13]. The majority of the methods for stability analysis and control design of time delay systems do not handle the nonnegativity of the state variables, so these methods can only limitedly use to the stabilization of nonnegative systems. An exception is the case of linear

\footnotetext{
*This research has been supported by the Hungarian National Research, Development and Innovation Office - NKFIH through the grant K115694. The work has also been supported by the European Union, co-financed by the European Social Fund through the grant EFOP-3.6.3-VEKOP-162017-00002. The work has also been supported by the Higher Education Excellence Program of the Ministry of Human Capacities in the frame of Artificial Intelligence research area of Budapest University of Technology and Economics (BME FIKPMI/FM).

${ }^{1}$ Process Control Research Group, Systems and Control Laboratory, Institute for Computer Science and Control (MTA SZTAKI), Hungarian Academy of Sciences, Kende u. 13-17, H1111 Budapest, Hungary lipgyorgyascl.sztaki.hu, hangosescl.sztaki.hu, szederesztaki.hu

${ }^{2}$ Department of Electrical Engineering and Information Systems, University of Pannonia, Egyetem u. 10, H-8200 Veszprém, Hungary

${ }^{3}$ Faculty of Information Technology and Bionics, Pázmány Péter Catholic University, Práter u. 50/a, H-1083 Budapest, Hungary
}

systems, where in [5], [19] stabilization methods for linear nonnegative systems are presented.

The class of kinetic systems, for which important and useful results are known on the relation between the dynamical properties and the associated graph structure, is useful for modeling a wide range of processes in various application fields, where the state variables are nonnegative [4]. Beside the ability to describe complex nonlinear phenomena, kinetic systems have a simple mathematical structure facilitating the kinetic realization of nonlinear models and the application of computational methods for model analysis and controller design. The original goal for using delays in kinetic models was to reduce the number of state variables by omitting certain intermediates and thus to focus on the most important species [24]. In [8] it shown that the memory effect caused by the complex environment of a biochemical network can be described by introducing an appropriate delay. It is proved in [3] that a class of time-varying stochastic delays in reaction networks can be exactly modeled by introducing a series of so-called delay species and delay reactions.

A key property of kinetic systems is called complex balance guaranteeing stability, which is global with a known parameter-independent logarithmic Lyapunov function in several special cases, and possibly even generally [6]. The complex balance property has been defined and analysed recently for kinetic systems containing constant time delays in [15], where it was shown using an appropriate LyapunovKrasovskii functional that complex balanced kinetic systems with arbitrary constant time-delays are semistable.

The directed graph structure and parametrization (called realization) of a polynomial kinetic system is generally non-unique, and complex balance (among other important features) is a realization property [17]. Utilizing this fact, an optimization based feedback design method was proposed in [18] to transform a nonlinear polynomial model to a closed loop system which has a complex balanced realization. Motivated by this, the purpose of this paper is to propose a solution for the stabilizing controller design problem in the kinetic framework for delayed nonnegative polynomial models.

\section{PReliminaries AND notations}

Throughout the paper, we will use the following notations. If $n$ is a positive integer, $\mathbb{R}^{n}$ and $\mathbb{Z}^{n}$ denote the $n$-dimensional space of real and integer column vectors, respectively. The symbols $\mathbb{R}_{+}^{n}\left(\mathbb{Z}_{+}^{n}\right)$ and $\overline{\mathbb{R}}_{+}^{n}\left(\overline{\mathbb{Z}}_{+}^{n}\right)$ denote the set of (element-wise) positive and nonnegative real (integer) vectors, respectively. For every $\tau \geq 0$, the symbol 
$\mathcal{C}=C\left([-\tau, 0], \mathbb{R}^{n}\right)$ denotes the Banach space of continuous functions mapping the interval $[-\tau, 0]$ into $\mathbb{R}^{n}$ with the norm $\|\phi\|=\sup _{-\tau \leq s \leq 0}|\phi(s)|$ for $\phi \in \mathcal{C}$, where $|\cdot|$ denotes the Euclidean norm in $\mathbb{R}^{n}$. Let $\mathcal{C}_{+}=C\left([-\tau, 0], \mathbb{R}_{+}^{n}\right)$ and $\overline{\mathcal{C}}_{+}=C\left([-\tau, 0], \overline{\mathbb{R}}_{+}^{n}\right)$ denote the set of positive and nonnegative functions in $\mathcal{C}$. For an $n$-dimensional column vector $v, \operatorname{diag}(v)$ is the $n \times n$ diagonal matrix with $v_{1}, \ldots, v_{n}$ in its diagonal. Finally, 1 denotes a column vector with all entries are 1 , and $\mathbf{0}$ denotes the zero vector.

\section{A. Nonnegative polynomial systems with time delay}

We consider a continuous-time polynomial autonomous system with time delay in the following form

$$
\dot{x}(t)=F\left(x_{t}\right)=M_{0} \psi(x(t))+\sum_{i=1}^{p} M_{i} \psi\left(x\left(t-\tau_{i}\right)\right),
$$

where $x(t) \in \mathbb{R}^{n}$ is the state of the system, $M_{i} \in \mathbb{R}^{n \times m}$, $i=0, \ldots, p$, and $\tau_{i}>0$ are the time delays. The monomial mapping $\psi$ is defined as

$$
\psi_{j}(x)=\prod_{i=1}^{n} x_{i}^{Y_{i j}}, \quad j=1, \ldots, m,
$$

where the exponents $Y_{i j}$ are nonnegative integers forming the monomial composition matrix $Y \in \overline{\mathbb{Z}}_{+}^{n \times m}$.

Solutions of (1) are generated by initial data $x(t)=\phi(t)$ for $-\tau \leq t \leq 0$, where $\tau$ is the largest delay and $\phi \in \overline{\mathcal{C}}_{+}$is a nonnegative continuous vector valued initial function. The solution of (1) with initial function $\phi \in \overline{\mathcal{C}}_{+}$will be denoted by $x=x^{\phi}$. For every $t \geq 0$, a segment of the solution is defined by $x_{t}(s)=x(t+s)$ for $-\tau \leq s \leq 0$.

A delayed differential equation system is called nonnegative when its solutions are nonnegative for every nonnegative initial function. The time delayed polynomial system (1) is nonnegative if and only if the following condition is fulfilled [25]: if $\phi \in \overline{\mathcal{C}}_{+}$and $\phi_{i}(0)=0$ for some $i \in\{1, \ldots, n\}$, then $F_{i}(\phi) \geq 0$. This condition is equivalent to that the matrices $M_{1}, \ldots, M_{p}$ are nonnegative and $M_{0} \psi(x)$ is essentially nonnegative i.e. $\left[M_{0} \psi(x)\right]_{i} \geq 0$ for all $i=1, \ldots, n$, and $x \in \overline{\mathbb{R}}_{+}^{n}$ such that $x_{i}=0$.

\section{B. Time delayed kinetic systems}

Kinetic systems: [4] form a special subclass of nonnegative polynomial systems. The description of a kinetic system is based upon the notion of species $X_{1}, X_{2} \ldots, X_{n}$, complexes $C_{1}, C_{2}, \ldots, C_{m}$ and reactions between the complexes. The complexes, that correspond to the monomials above, are defined by the linear nonegative integer combination of the species, i.e $C_{j}=\sum_{i=1}^{n} Y_{i, j} X_{i}$ for $j=1, \ldots, m$. The dynamic model of a kinetic system describes the transformation of the complexes, into each other in the form of reactions $C_{i} \mapsto C_{j}$. A positive constant $\kappa_{i, j}$ is associated to each reactions as a reaction rate coefficient.

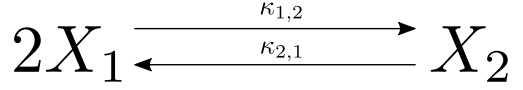

Fig. 1. Reaction graph of the example model (4)

Reaction graph: Similarly to [9] and many other authors, we can represent the set of individual reaction steps by a weighted directed graph called reaction graph. The reaction graph consists of a set of vertices and a set of directed edges. The vertices correspond to the complexes, while the directed edges represent the reactions, i.e. if we have a reaction $C_{i} \mapsto C_{j}$ then there is an edge in the reaction graph between the complexes $C_{i}$ and $C_{j}$ with the weight $\kappa_{i, j}$.

It is important to note that the notion of zero complex $C_{0}=\emptyset$ is used to describe open kinetic systems with constant input concentrations.

ODE model: The time evolution of a kinetic system can be described by an ordinary differential equation where the state variables $x(t) \in \overline{\mathbb{R}}_{+}^{n}$ are the concentrations of the species. The ODEs are defined as follows

$$
\dot{x}(t)=Y\left[A-\operatorname{diag}\left(\mathbf{1}^{T} A\right)\right] \psi(x(t)),
$$

where $\psi$ is the monomial mapping (2) generated by $Y \in$ $\overline{\mathbb{Z}}_{+}^{n \times m}$. The matrix $A \in \overline{\mathbb{R}}_{+}^{n \times n}$ is a nonnegative and contains the reaction coefficients, i.e. $A_{i, j}=\kappa_{j, i}$.

Simple undelayed example: Consider two species, $X_{1}$ and $X_{2}$ that react in a reversible reaction $2 X_{1} \leftrightarrows X_{2}$. Then the elementary reaction steps are

$$
2 X_{1} \stackrel{\kappa_{1,2}}{\longrightarrow} X_{2} ; \quad X_{2} \stackrel{\kappa_{2,1}}{\longrightarrow} 2 X_{1},
$$

where the complexes are $C_{1}=2 X_{1}$ and $C_{2}=X_{2}$. The corresponding reaction graph is depicted in Fig. 1. The ODEs (3) have the following parameters

$$
Y=\left[\begin{array}{ll}
2 & 0 \\
0 & 1
\end{array}\right], A=\left[\begin{array}{cc}
0 & \kappa_{2,1} \\
\kappa_{1,2} & 0
\end{array}\right], \psi(x)=\left[\begin{array}{l}
x_{1}^{2} \\
x_{2}
\end{array}\right] .
$$

Time delayed kinetic systems: We can extend the undelayed kinetic system (3) with $p$ different time delays $\tau_{1}, \tau_{2}, \ldots \tau_{p}$ in such a way, that each reaction has also a nonnegative real number associated to it that represents the time delay of the reaction. Then, we can describe the dynamics in the following form

$$
\begin{aligned}
\dot{x}(t)= & Y\left[A_{0}-\sum_{i=0}^{p} \operatorname{diag}\left(\mathbf{1}^{T} A_{i}\right)\right] \psi(x(t)) \\
& +\sum_{i=1}^{p} Y A_{i} \psi\left(x\left(t-\tau_{i}\right)\right),
\end{aligned}
$$

where $\psi$ is the monomial mapping (2). The nonnegative matrix $A_{0}$ contains the reaction rates of the undelayed reactions and the nonnegative matrices $A_{1}, \ldots, A_{p}$ contain the reaction rate coefficients of the reactions which are delayed by $\tau_{1}, \ldots, \tau_{p}$, respectively. 


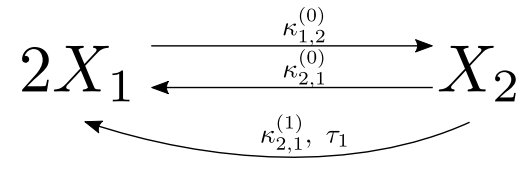

Fig. 2. Reaction graph of the example model (6)

Reaction graph with time delay: We can simply extend the reaction graph of a kinetic system with time delays. In this case, it is a directed and labeled multigraph, where the label of an edge is not only the reaction rate constant, but also the time delay. Reactions with same source and product complexes, but different time delays occur as parallel edges in the reaction graph.

Delayed example: Consider again two species, $X_{1}$ and $X_{2}$ that react in a reversible reaction $2 X_{1} \leftrightarrows X_{2}$, and let us have a third reaction converting $X_{2}$ to $2 X_{1}$ with a delay. Then the elementary reaction steps are

$$
2 X_{1} \stackrel{\kappa_{1,2}^{(0)}}{\longrightarrow} X_{2} ; X_{2} \stackrel{\kappa_{2,1}^{(0)}}{\longrightarrow} 2 X_{1} ; X_{2} \stackrel{\kappa_{2,1}^{(1)}, \tau_{1}}{\longrightarrow} 2 X_{1}
$$

The corresponding reaction graph is depicted in Fig. 2. The DDEs (5) have the following parameters

$$
\begin{array}{r}
Y=\left[\begin{array}{ll}
2 & 0 \\
0 & 1
\end{array}\right], \psi(x)=\left[\begin{array}{c}
x_{1}^{2} \\
x_{2}
\end{array}\right], \\
A_{0}=\left[\begin{array}{cc}
0 & \kappa_{2,1}^{(0)} \\
\kappa_{1,2}^{(0)} & 0
\end{array}\right], A_{1}=\left[\begin{array}{cc}
0 & \kappa_{2,1}^{(1)} \\
0 & 0
\end{array}\right] .
\end{array}
$$

Time delayed kinetic realizations: We say that the time delayed system (1) is kinetically realizable (or shortly, kinetic) with the monomial composition matrix $Y$ if it can be represented in the form (5), i.e. there exist $p+1$ nonnegative matrices $A_{0}, \ldots, A_{p}$ such that

$$
\begin{aligned}
M_{0} & =Y\left[A_{0}-\sum_{i=0}^{p} \operatorname{diag}\left(\mathbf{1}^{T} A_{i}\right)\right], \\
M_{i} & =Y A_{i}, \quad i=1, \ldots, p .
\end{aligned}
$$

The tuple of matrices $\left(Y, A_{0}, \ldots, A_{p}\right)$ is called a kinetic realization.

It is easy to verify that the existence of the factorization in Eq. (7) allows the direct construction of the reaction graph of a delayed nonnegative system as it is defined in [15]. Moreover, if $Y$ is fixed then (7) is a linear constraint and thus it can be solved (non-uniquely in general) for $A_{0}, \ldots, A_{p}$ e.g. in the framework of linear programming.

Remark: When a monomial introduced by $Y$ does not occur explicitly in Eq. (1) then the corresponding column of matrices $M_{0}, \ldots, M_{p}$ is zero.

C. Stability of complex balanced kinetic systems with time delay

Let us define the matrices $M=\sum_{i=0}^{p} M_{i}$ and $A=$ $\sum_{i=0}^{p} A_{i}$. Then we obtain the undelayed kinetic system corresponding to (5) as follows

$$
\dot{x}(t)=M \psi(x(t))=Y\left[A-\operatorname{diag}\left(\mathbf{1}^{T} A\right)\right] \psi(x(t)),
$$

where the matrix $A-\operatorname{diag}\left(\mathbf{1}^{T} A\right)$ is a column conservation matrix (or a Kirchhoff matrix).

Clearly, the positive vector $x^{*} \in \mathbb{R}_{+}^{n}$ is an equilibrium point of the time delayed kinetic system if and only if it is an equilibrium point of the corresponding undelayed kinetic system (8), i.e.

$$
Y\left[A-\operatorname{diag}\left(\mathbf{1}^{T} A\right)\right] \psi\left(x^{*}\right)=\mathbf{0} .
$$

The positive equilibrium point $x^{*}$ is called complex balanced if it fulfils the equation [14]

$$
\left[A-\operatorname{diag}\left(\mathbf{1}^{T} A\right)\right] \psi\left(x^{*}\right)=\mathbf{0} .
$$

We call a kinetic system complex balanced with or without time delay, if the complex balanced property is confirmed for any equilibrium point $x^{*}$.

Remark: A kinetic system may have a connected set of equilibria, therefore we introduce the following definition. An equilibrium $x^{*}$ of Eq. (5) is called semistable if it is Lyapunov stable and there exists $\delta>0$ such that if $\phi \in \mathcal{C}$ and $\left\|\phi-x^{*}\right\| \leq \delta$, then $x^{\phi}(t)$ converges to a Lyapunov stable equilibrium of (5) as $t \rightarrow \infty$. For more details on the notion and application of semistability, see [11].

We will use the following recent stability result for complex balanced time delayed kinetic systems.

Theorem 2.1 ([15]): Every complex balanced equilibrium $x^{*}$ of the delayed kinetic system (5) is semistable. Moreover, when it has only one complex balanced equilibrium point, then it is locally asymptotically stable with respect to the positive orthant.

\section{Unique complex balanced equilibrium}

In this subsection, we give a necessary and sufficient condition for the unique solution of Eq. (9). For this purpose, the Laplacian matrix $\mathcal{L}\left(x^{*}\right)$ at a positive equilibrium point is introduced based on [27] as

$$
\mathcal{L}\left(x^{*}\right)=\left[\operatorname{diag}\left(\mathbf{1}^{T} A\right)-A\right] \operatorname{diag}\left(\psi\left(x^{*}\right)\right) .
$$

Then, the positive equilibrium point $x^{*}$ is complex balanced if and only if the Laplacian $\mathcal{L}\left(x^{*}\right)$ is balanced, i.e $\mathcal{L}\left(x^{*}\right) \mathbf{1}=$ 0 .

This gives rise to our uniqueness result below.

Theorem 2.2 ([16]): Let the equilibrium $x^{*}$ be complex balanced. Then, $x^{*}$ is unique positive equilibrium if and only if the matrix

$$
Y\left[\mathcal{L}\left(x^{*}\right)+\mathcal{L}\left(x^{*}\right)^{T}\right] Y^{T}
$$

is positive definite.

\section{FORMULATION AND SOLUTION OF THE FEEDBACK DESIGN PROBLEM}

In this section, we consider a stabilizing feedback design method for the open loop system

$$
\dot{x}(t)=M_{0} \psi(x(t))+\sum_{i=1}^{p} M_{i} \psi\left(x\left(t-\tau_{i}\right)\right)+B u(t),
$$

where $u(t) \in \mathbb{R}^{r}$ is the input of the system, and $B \in \mathbb{R}^{n \times r}$. 
It is important to note that the above open loop system (11) is not necessarily kinetic, but it is a delayed polynomial system with LTI input structure. In the special case when the open system (11) is kinetic, its model corresponds to an ODE model of an open delayed CRN where the inputs are chosen as the concentrations of the species in the inlet flows, see the discussion in [18] of the non-delayed case.

The aim of the feedback is to stabilize the open loop system (11) locally asymptotically in the given positive equilibrium point $x^{*}$. For this, we transform the open loop system (11) into a complex balanced kinetic closed loop system with a desired equilibrium point $x^{*}$. The problem will be formulated as a semidefinite programming (SDP) problem.

\section{A. The state feedback law}

We assume a polynomial feedback

$$
u(t)=K_{0} \psi(x(t))+\sum_{i=1}^{p} K_{i} \psi\left(x\left(t-\tau_{i}\right)\right),
$$

where the matrices $K_{i} \in \mathbb{R}^{r \times m}, i=0, \ldots, p$ are the parameters of the feedback. Then, the closed loop system has the form

$$
\begin{aligned}
\dot{x}(t)= & {\left[M_{0}+B K_{0}\right] \psi(x(t)) } \\
& +\sum_{i=1}^{p}\left[M_{i}+B K_{i}\right] \psi\left(x\left(t-\tau_{i}\right)\right) .
\end{aligned}
$$

\section{B. Feedback computation}

We are looking for the feedback parameters $K_{0}, \ldots, K_{p}$ such that the closed-loop system (13) has a kinetic realization $\left(Y, A_{0}, \ldots, A_{p}\right)$ which has the unique complex balanced equilibrium $x^{*}$. Therefore, the decision variables are $K_{i} \in$ $\mathbb{R}^{r \times m}$, and $A_{i} \in \overline{\mathbb{R}}_{+}^{m \times m}, i=0, \ldots, p$.

The first constraint is used to guarantee that the solution will be a kinetic realization of the closed-loop system. It is linear in the decision variables and has the form

$$
\begin{aligned}
M_{0}+B K_{0} & =Y\left[A_{0}-\sum_{i=0}^{p} \operatorname{diag}\left(\mathbf{1}^{T} A_{i}\right)\right], \\
M_{i}+B K_{i} & =Y A_{i}, \quad i=1, \ldots, p .
\end{aligned}
$$

The closed loop system should be complex balanced, which is ensured by the linear constraint

$$
\left[A-\operatorname{diag}\left(\mathbf{1}^{T} A\right)\right] \psi\left(x^{*}\right)=\mathbf{0},
$$

where $A=\sum_{i=0}^{p} A_{i}$.

Finally, the uniqueness of the equilibrium point $x^{*}$ is guaranteed by the positive definite constraint

$$
Y\left[\mathcal{L}\left(x^{*}\right)+\mathcal{L}\left(x^{*}\right)^{T}\right] Y^{T}>0,
$$

where $\mathcal{L}\left(x^{*}\right)$ is the Laplacian matrix (10) in the given equilibrium point $x^{*}$.
The optimization problem: By putting together the constraints described in (14)-(16) and considering the $l_{1}$ norm of the feedback parameters as an objective function, the semidefinite programming problem can be constructed:

$$
\begin{aligned}
\min _{K_{i}, A_{i}} & \sum_{i=0}^{p} \sum_{j=1}^{r} \sum_{k=1}^{m}\left|\left[K_{i}\right]_{j, k}\right|, \\
\text { s.t. } \quad & M_{0}+B K_{0}=Y\left[A_{0}-\sum_{i=0}^{p} \operatorname{diag}\left(\mathbf{1}^{T} A_{i}\right)\right], \\
& M_{i}+B K_{i}=Y A_{i}, \quad i=1, \ldots, p, \\
& {\left[A-\operatorname{diag}\left(\mathbf{1}^{T} A\right)\right] \psi\left(x^{*}\right)=\mathbf{0}, } \\
& Y\left[\mathcal{L}\left(x^{*}\right)+\mathcal{L}\left(x^{*}\right)^{T}\right] Y^{T}>0,
\end{aligned}
$$

where $A=\sum_{i=0}^{p} A_{i}$ and $x^{*}>0$ is given.

\section{Remarks:}

(R1) When the uniqueness of the equilibrium point is not required, then we can compute the stabilizing feedback using only the constraints (14)-(15) via linear programming.

(R2) In the optimization problem (17), we use an objective function which computes sparse feedback matrices $K_{i}$. This is not the only possible choice, e.g. in the paper [18], we proposed an objective function which minimizes the eigenvalue of the linearized closed-loop system with the largest real part.

(R3) The optimization problem is linear in the feedback parameters $K_{i}$, so we can introduce additional constraints for the feedback structure, e.g. we can prescribe a distributed control structure, or exclude some delays from the feedback.

\section{ILLUSTRATIVE EXAMPLE}

In the following, we present the applicability of the proposed design technique on an illustrative example. The algorithms were implemented in [21] using the YALMIP modelling language [20]. Mosek [1] was used to solve the SDP problems.

\section{A. System description}

Let us consider the nonnegative open loop system in the form

$$
\begin{aligned}
& \dot{x}_{1}(t)=2 x_{1}^{2}(t)-2 x_{1}(t) x_{2}(t)+u(t) \\
& \dot{x}_{2}(t)=-x_{2}(t)+x_{1}^{2}\left(t-\tau_{1}\right),
\end{aligned}
$$

where $x_{1}(t), x_{2}(t)$ are the states, $u(t)$ is the input and $\tau_{1}>0$ is an arbitrary constant time delay. The open loop system is characterized by the following matrices

$$
\begin{gathered}
M_{0}=\left[\begin{array}{rrr}
0 & 2 & -2 \\
-1 & 0 & 0
\end{array}\right], \quad M_{1}=\left[\begin{array}{lll}
0 & 0 & 0 \\
0 & 1 & 0
\end{array}\right], \\
Y=\left[\begin{array}{lll}
0 & 2 & 1 \\
1 & 0 & 1
\end{array}\right], \quad B=\left[\begin{array}{l}
1 \\
0
\end{array}\right] .
\end{gathered}
$$

For $u(t)=0$, the system has an unstable positive equilibrium point in $x_{1}^{*}=x_{2}^{*}=1$. 


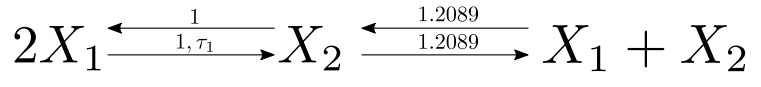

Fig. 3. Reaction graph of the closed loop realization (20)

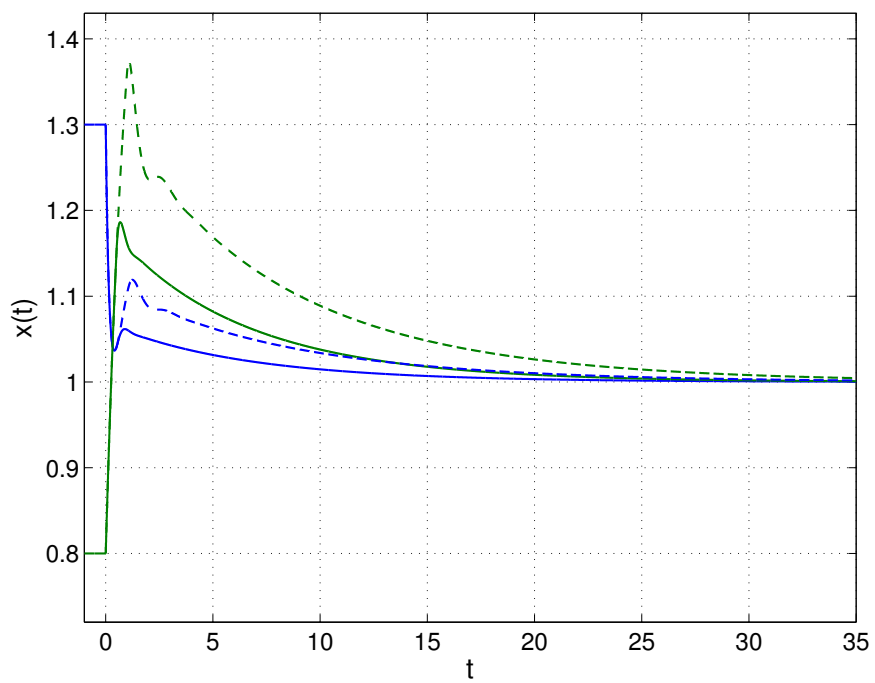

Fig. 4. Simulation results for the system (18) with the stabilizing feedback (19). The simulation is started from two different constant initial functions, $\phi_{1}(s)=1.3$, and $\phi_{2}(s)=0.8$ with two different time delays $\tau_{1}=0.5$, and $\tau_{1}=1$. The blue and green curves show the simulated states $x_{1}$ and $x_{2}$, respectively. The dashed-curves correspond to the larger delay $\tau_{1}=1$

\section{B. Stabilizing feedback design}

Because of the unstable positive equilibrium point, the aim of the feedback is to stabilize this equilibrium point. In this case, we extend the original optimization problem (17) by an additional constraint to exclude the delay element from the feedback. Then the optimization results the following feedback parameters

$$
K_{0}=\left[\begin{array}{lll}
3.2089 & -4 & 0.7911
\end{array}\right], \quad K_{1}=\left[\begin{array}{lll}
0 & 0 & 0
\end{array}\right],
$$

and therefore, the input is computed as

$$
u(t)=3.2089 x_{2}(t)-4 x_{1}^{2}(t)+0.7911 x_{1}(t) x_{2}(t) .
$$

The computed feedback (19) does not contain delay. The computed kinetic realization of the closed loop system is given by

$$
A_{0}=\left[\begin{array}{rrr}
0 & 0 & 1.209 \\
1 & 0 & 0 \\
1.209 & 0 & 0
\end{array}\right], A_{1}=\left[\begin{array}{lll}
0 & 1 & 0 \\
0 & 0 & 0 \\
0 & 0 & 0
\end{array}\right],
$$

which is complex balanced at the equilibrium point $x_{1}^{*}=$ $x_{2}^{*}=1$, so the closed loop system is stable. The stability is guaranteed by the following Lyapunov-Krasovskii functional [15]

$$
\begin{array}{r}
V\left(x_{t}\right)=x_{1}(t)\left[\ln \left(x_{1}(t)\right)-1\right]+x_{2}(t)\left[\ln \left(x_{2}(t)\right)-1\right]+2 \\
+\int_{t-\tau_{1}}^{t}\left\{x_{1}^{2}(s)\left[\ln \left(x_{1}^{2}(s)\right)-1\right]+1\right\} d s .
\end{array}
$$

Fig. 3 shows the reaction graph of the closed loop system and Fig. 4 illustrates the time domain behavior.

\section{DISCUSSION}

A feedback design method was proposed in this paper to transform a delayed polynomial system with linear input structure to a kinetic system having the complex balanced property which guarantees stability. The computability of the feedback does not depend on the magnitude of the delays. Moreover, the optimization-based computation framework allows the introduction of additional design constraints such as the exclusion of delayed monomials from the feedback.

Further work will be focused on the problem of new monomial selection in the feedback and on extending our method to handle parametric uncertainty, similarly to our previous results [18]. Furthermore, we plan to relate our stability conditions to other existing results such as [2] and [7] that were derived for different delayed model classes.

\section{REFERENCES}

[1] MOSEK ApS. The MOSEK optimization toolbox for MATLAB manual. Version 8.1., 2017.

[2] C. Briat. Stability and performance analysis of linear positive systems with delays using input-output methods. International Journal of Control, 91:1669-1692, 2018.

[3] C. Briat and M. Khammash. Ergodicity analysis and antithetic integral control of a class of stochastic reaction networks with delays. arXiv:1811.09188 [math.OC], 2018.

[4] V. Chellaboina, S. P. Bhat, W. M. Haddad, and D. S. Bernstein. Modeling and analysis of mass-action kinetics - nonnegativity, realizability, reducibility, and semistability. IEEE Control Systems Magazine, 29:60-78, 2009.

[5] V. Chellaboina, W.M. Haddad, J. Ramakrishnan, and T. Hayakawa. Direct adaptive control of nonnegative and compartmental dynamical systems with time delay. In Proceedings of the 2004 American Control Conference. IEEE, 2004.

[6] G. Craciun. Toric differential inclusions and a proof of the global attractor conjecture. arXiv:1501.02860 [math.DS], January 2015.

[7] E. Devane and I. Lestas. Delay-independent asymptotic stability in monotone systems. IEEE Transactions on Automatic Control, 61:2625-2631, 2015.

[8] J. Falk, L. Bronstein, M. Hanst, B. Drossel, and H. Koeppl. Context in synthetic biology: Memory effects of environments with monomolecular reactions. arXiv:1809.09555 [q-bio.MN], 2018.

[9] M. Feinberg. Lectures on chemical reaction networks. Notes of lectures given at the Mathematics Research Center, University of Wisconsin, 1979.

[10] Giuseppe Franze, Domenico Famularo, and Alessandro Casavola. Constrained nonlinear polynomial time-delay systems: A sum-ofsquares approach to estimate the domain of attraction. IEEE Transactions on Automatic Control, 57(10):2673-2679, oct 2012.

[11] Wassim M. Haddad. Nonnegative and Compartmental Dynamical Systems. Princeton University Press, 2010.

[12] J. K. Hale and S. M. Verduyn Lunel. Introduction to Functional Differential Equations. Springer, New York, 1993.

[13] K.M. Hangos and I.T. Cameron. Process modelling and model analysis. Academic Press, London, 2001.

[14] F. Horn and R. Jackson. General mass action kinetics. Archive for Rational Mechanics and Analysis, 47:81-116, 1972.

[15] Gy. Lipták, M. Pituk, K. M. Hangos, and G. Szederkényi. Semistability of complex balanced kinetic systems with arbitrary time delays. Systems \& Control Letters, 114:38-43, 2018.

[16] Gy. Lipták, J. Rudan, K. M. Hangos, and G. Szederkényi. Stabilizing kinetic feedback design using semidefinite programming. In 2nd IFAC Workshop on Thermodynamic Foundation of Mathematical Systems Theory - TFMST 2016, 09. 28 - 09. 30, 2016, Vigo, Spain, IFACPapersOnLine, volume 49, pages 12 - 17, 2016.

[17] Gy. Lipták, G. Szederkényi, and K. M. Hangos. Computing zero deficiency realizations of kinetic systems. Systems \& Control Letters, 81:24-30, 2015.

[18] Gy. Lipták, G. Szederkényi, and M. Hangos. Kinetic feedback design for polynomial systems. Journal of Process Control, 41:56-66, 2016. 
[19] Xingwen Liu. Constrained control of positive systems with delays. IEEE Transactions on Automatic Control, 54(7):1596-1600, jul 2009.

[20] J. Löfberg. YALMIP : A toolbox for modeling and optimization in MATLAB. In Proceedings of the CACSD Conference, Taipei, Taiwan, 2004.

[21] MATLAB. version 8.3.0 (R2014a). The MathWorks Inc., Natick, Massachusetts, 2014.

[22] A. Papachristodoulou. Robust stabilization of nonlinear time delay systems using convex optimization. In Proceedings of the 44th IEEE Conference on Decision and Control. IEEE, 2005.

[23] Jean-Pierre Richard. Time-delay systems: an overview of some recent advances and open problems. Automatica, 39(10):1667-1694, oct 2003.

[24] Marc R Roussel. The use of delay differential equations in chemical kinetics. The Journal of Physical Chemistry, 100(20):8323-8330, 1996.

[25] H. L. Smith. Monotone Dynamical Systems. An Introduction to the Theory of Competitive and Cooperative Systems, volume 41 of Mathematical Surveys and Monographs. American Mathematical Society, Providence, Rhode Island, 1995.

[26] Y. Takeuchi. Global Dynamical Properties of Lotka-Volterra Systems. World Scientific, Singapore, 1996.

[27] A. van der Schaft, S. Rao, and B. Jayawardhana. Complex and detailed balancing of chemical reaction networks revisited. Journal of Mathematical Chemistry, 53(6):1445-1458, 2015.

[28] P. Érdi and J. Tóth. Mathematical Models of Chemical Reactions. Theory and Applications of Deterministic and Stochastic Models. Manchester University Press, Princeton University Press, Manchester, Princeton, 1989. 